\title{
Diagnostic role of 18F-FDG PET/MRI in the TNM staging of breast cancer: a systematic review and meta-analysis
}

\author{
Xing-Ru Lu, Meng-Meng Qu, Ya-Nan Zhai, Wen Feng, Ya Gao, Jun-Qiang Lei \\ Department of Radiology, First Hospital of Lanzhou University, Lanzhou, China \\ Contributions: (I) Conception and design: XR Lu, JQ Lei; (II) Administrative support: XR Lu, JQ Lei; (III) Provision of study materials or patients: \\ XR Lu, MM Qu, YN Zhai; (IV) Collection and assembly of data: XR Lu, W Feng, Y Gao; (V) Data analysis and interpretation: XR Lu, YN Zhai, \\ MM Qu; (VI) Manuscript writing: All authors; (VII) Final approval of manuscript: All authors. \\ Correspondence to: Jun-Qiang Lei. Department of Radiology, First Hospital of LanZhou University, No. 1, Donggang West Road, Chengguan \\ District, Lanzhou 730000, China. Email: leijq2011@sina.com.
}

\begin{abstract}
Background: To investigate the value of $18 \mathrm{~F}$-fluorodeoxyglucose positron emission tomography/magnetic resonance imaging (18F-FDG PET/MRI) in diagnosing local tumor invasion ( $\mathrm{T}$ stage), evaluating regional lymph node involvement ( $\mathrm{N}$ stage), and detecting distant metastasis ( $\mathrm{M}$ stage) in breast cancer patients.

Methods: A comprehensive computer search and manual search were performed to select any potentially eligible studies that evaluated the diagnostic efficacy of 18F-FDG PET/MRI in the tumor-node-metastasis (TNM) staging of breast cancer. Data from the included studies were extracted to calculate the pooled sensitivity, specificity, and area under the curve (AUC) to evaluate the value of 18F-FDG PET/MRI in TNM staging. Quality and publication bias were also assessed in this meta-analysis.

Results: Based on seven studies, the pooled sensitivity, specificity and AUC for the diagnosis of T stage were 91\% (95\% CI: 84-96\%), 91\% (95\% CI: 81-96\%), and 0.96 (95\% CI: 0.94-0.98), respectively. For $\mathrm{N}$ stage evaluation, four studies were included, with values of 94\% (95\% CI: 83-98\%), 90\% (95\% CI: 81$95 \%$ ), and 0.96 (95\% CI: 0.94-0.97). For M stage detection, five studies were evaluated, with values of $98 \%$ (95\% CI: 96-99\%), 96\% (95\% CI: 83-99\%), and 0.99 (95\% CI: 0.98-1.00).

Conclusions: 18F-FDG PET/MRI demonstrates higher diagnostic value in the TNM staging of breast cancer and can serve as an effective and promising imaging biomarker for future evaluation of TNM stage in breast cancer patients.
\end{abstract}

Keywords: Breast cancer; 18F-fluorodeoxyglucose positron emission tomography/magnetic resonance imaging (18F-FDG PET/MRI); tumor-node-metastasis stage (TNM stage); meta-analysis

Submitted Dec 21, 2020. Accepted for publication Mar 05, 2021.

doi: 10.21037/apm-20-2555

View this article at: http://dx.doi.org/10.21037/apm-20-2555

\section{Introduction}

Breast cancer is the most frequently diagnosed cancer and is also the leading cause of cancer-related death among women worldwide, accounting for almost $25 \%$ of cancer cases and more than $14.7 \%$ of cancer-related deaths in this population $(1,2)$. Surgical treatment followed by neoadjuvant therapy is optimal for local breast cancer invasion with or without regional lymph node involvement and has been proven to prolong the 5 -year survival rate to $85-98 \%$ (3). However, patients with distant metastasis only receive neoadjuvant treatment and have a poor 5 -year survival rate of $30-70 \%$ (4). Therefore, accurate initial breast cancer staging, including diagnosis of local tumor invasion ( $\mathrm{T}$ stage), evaluation of regional lymph node involvement ( $\mathrm{N}$ stage), and detection of distant metastases (M stage) — known as TNM staging (5) - is mandatory to guide optimal therapeutic treatment and predict patient prognosis.

18F-fluorodeoxyglucose positron emission tomography/ 
magnetic resonance imaging (18F-FDG PET/MRI) is a radiation-free imaging modality that combines the metabolic information of FDG PET with the highresolution soft tissue characteristics of $M R I$ in a single 18F-FDG PET/MRI examination $(6,7)$. An increasing number (6-17) of studies have demonstrated that 18F-FDG $\mathrm{PET} / \mathrm{MRI}$ is a valuable alternative imaging method in breast cancer staging because it provides a potentially promising one-stop-shop examination for patients with breast cancer who should undergo both breast MRI for local tumor invasion diagnosis/lymph node detection and whole-body PET for distant metastasis evaluation.

A meta-analysis published in 2018 aimed to assess the whole-body staging/restaging accuracy of 18F-FDG PET/ MRI in breast cancer (18). However, this study mixed TNM staging together into whole-body staging/restaging and did not evaluate the differences in diagnostic accuracy for T stage, $\mathrm{N}$ stage, and $\mathrm{M}$ stage independently. Additionally, several studies that evaluated the value of $18 \mathrm{~F}-\mathrm{FDG}$ PET/ MRI in breast cancer staging/restaging were neglected in their study $(6,7,11,14,15)$, which may reduce the precision of this research result.

Therefore, this meta-analysis was firstly performed to comprehensively assess the value of 18F-FDG PET/ MRI in assessing TNM stage in breast cancer to our best knowledge, hoping to provide evidence-based imaging biomarkers for guiding clinical treatment and predicting prognosis in breast cancer patients.

We present the following article in accordance with the PRISMA reporting checklist. available at: http://dx.doi. org/10.21037/apm-20-2555.

\section{Methods}

\section{Data sources and literature search strategy}

This study was performed with reference to the Preferred Reporting Items for Systematic reviews and MetaAnalyses (PRISMA) guidelines (19). A comprehensive literature search of the PubMed, Embase, Cochrane Library and Embase databases up to October 2020 was performed to explore original studies that evaluated the value of $18 \mathrm{~F}-\mathrm{FDG}$ PET/MRI in evaluating TNM stage in breast cancer patients using the combination of the following key words: (I) "positron emission tomography" or "PET"; and (II) "magnetic resonance imaging" or "MR"; and (III) "breast cancer". Then, a manual search of the included study references and identified review articles was performed to select any potentially eligible trials.

\section{Study selection}

\section{Inclusion criteria}

Studies were included if they met the following inclusion criteria: (I) the index test, 18F-FDG PET/MRI, was used to evaluate TNM stage in breast cancer patients; (II) pathology, follow-up imaging, or a combination of two methods served as the reference standard; (III) the numbers of true positives (TPs), false positives (FPs), false negatives (FNs) and true negatives (TNs) was presented in a fourfold table or could be extracted from the included study; and (IV) the last or the most comprehensive study was included when the data overlapped between studies.

\section{Exclusion criteria}

Studies were excluded according to the following criteria: (I) only PET or MRI examination was adopted to assess TNM stage in breast cancer patients; (II) whole-body staging/restaging, rather than TNM staging, performance of $18 \mathrm{~F}-\mathrm{FDG}$ PET/MRI in breast cancer patients was the focus; (III) fewer than ten patients with breast cancer underwent 18F-FDG PET/MRI evaluation; (IV) patients with other malignant tumors or hematological disease were included; or (V) studies were not in English or were review articles, conference abstracts or animal studies.

\section{Data extraction and quality assessment}

Data extraction and quality assessment were independently performed by two researchers (first author and second author), and any discrepancies were solved by discussion in consensus.

A predesigned Excel table was adopted to extract data from the included studies, including first author, origin country, publication year, prospective or retrospective study design, baseline characteristics (number, mean age, sex, number of breast lesions, and lesion size), reference standard, blinding method, fourfold table data (TP, FP, FN, $\mathrm{TN}$ ), and data type (patient-based or lesion-based).

The Quality Assessment of Diagnostic Accuracy Studies-2 (QUADAS-2) guidelines were applied to evaluate the methodological quality of risk bias and clinical applicability of the study from seven domains concerning the study characteristics. The bias risk or applicability concerns can be evaluated as low, unclear or high risk. Unclear risk indicates that the included study only partially 
meets the criteria or does not provide adequate information, while low risk and high risk indicate that the study satisfies or does not satisfy the criteria, respectively.

\section{Statistical analysis}

Heterogeneity among the included studies was assessed using the Cochran $\mathrm{Q}$ test $(\mathrm{P}<0.05$ was indicative of significant heterogeneity) and I-square statistic algorithms for TNM stage separately, and the degree of heterogeneity was classified as low $\left(\mathrm{I}^{2}=25-49 \%\right)$, moderate $\left(\mathrm{I}^{2}=50 \%\right.$ to $74 \%)$, and high $\left(\mathrm{I}^{2}>75 \%\right)$. To present the characteristics of the studies that applied 18F-FDG PET/MRI to evaluate breast cancer TNM stages appropriately, the summary sensitivity, specificity, diagnostic odds ratio (DOR), positive likelihood ratio (PLR), and negative likelihood ratio (NLR) with $95 \%$ confidence intervals (CIs) were calculated based on fourfold table values using bivariate random effects. Then, the area under the receiver operating characteristic (ROC) curve (AUC) was calculated by hierarchical regression to evaluate the efficacy of $18 \mathrm{~F}-\mathrm{FDG}$ PET/ MRI in evaluating TNM stages with breast cancer. The diagnostic value was regarded as good for AUC values $>0.9$ and medium for AUC values from 0.7 to 0.9 .

Moreover, subgroup analysis was predesigned to analyze potential factors, such as study design (retrospective or prospective) and data type (patient-based or lesionbased), that could contribute to heterogeneity in this study. Additionally, funnel plots were generated and evaluated with Deeks' asymmetry test to assess publication bias for 18F-FDG PET/MRI in the TNM staging of breast cancer, and a $\mathrm{P}$ value $<0.05$ was representative of publication bias. Statistical analysis was performed with Review Manager software (version 5.3, the Cochrane Collaboration, 2014) and STATA software (version 12.0, StataCorp, College Station, TX, USA).

\section{Results}

\section{Study selection and baseline characteristics}

A total of 2,164 potential studies were primarily investigated based on computer and manual searches, and 1,404 articles were retrieved after removing duplicates through Endnote X7 software. After screening the titles and abstracts, only 54 references were remined for full text review. Then, 42 articles not meeting the inclusion criteria were excluded, and 12 studies (6-17) that evaluated the diagnostic performance of 18F-FDG PET/MRI in breast cancer TNM staging were finally included in this meta-analysis. The detailed diagram of study selection process is shown in Figure 1.

Baseline characteristics extracted from each included study are presented in Table 1 . The twelve included studies were single-center studies comprising 666 patients and were conducted in seven countries and published between 2010 and 2019; seven were prospective studies and five were retrospective studies. Among the included studies, five studies diagnosed $\mathrm{T}$ stage $(6,7,11,13,14)$, one study evaluated $\mathrm{N}$ stage (17), three studies detected $M$ stage $(9,15,16)$, one study evaluated $\mathrm{T}$ stage and $\mathrm{N}$ stage (10), one study diagnose $\mathrm{N}$ stage and $\mathrm{M}$ stage (7), and one study assessed TNM stage (12).

\section{Methodological quality assessment}

Table 2 demonstrates the methodological quality assessment of the twelve studies. All included studies involved consecutive breast cancer patients who underwent 18F-FDG PET/MRI evaluation for TNM staging without a casecontrol design, and thus, all enrolled studies presented a low risk of bias in the domain of patient selection. Regarding the index test domain, four studies were classified as unclear risk owing to unclear blinding methods $(9,11,15,17)$. Pathology and/or follow-up are all reliable reference standards in the TNM staging of breast cancer patients in this meta-analysis; therefore, all twelve studies demonstrated a low risk of bias in the reference standard domain. The flow and timing domain evaluation was mainly associated with whether the same reference standard was adopted and whether there was an appropriate interval between the index test and reference standard. Five studies and 7 studies were considered to have high risk and unclear risk bias, respectively, due to unclear or longer time intervals between 18F-FDG PET/ MRI examination and the reference standard and different reference standard methods.

For applicability concerns regarding patient selection, all studies were regarded as having low risk bias due to the consecutive enrollment of patients and the uncontrolled designs. The applicability concern domain of the index test was low risk for the 12 studies that assessed the diagnostic value of $18 \mathrm{~F}-\mathrm{FDG}$ PET/MRI in breast cancer staging. In addition, reference standard applicability concerns presented low risk for all included studies, owing to clear pathological or follow-up imaging criteria. 


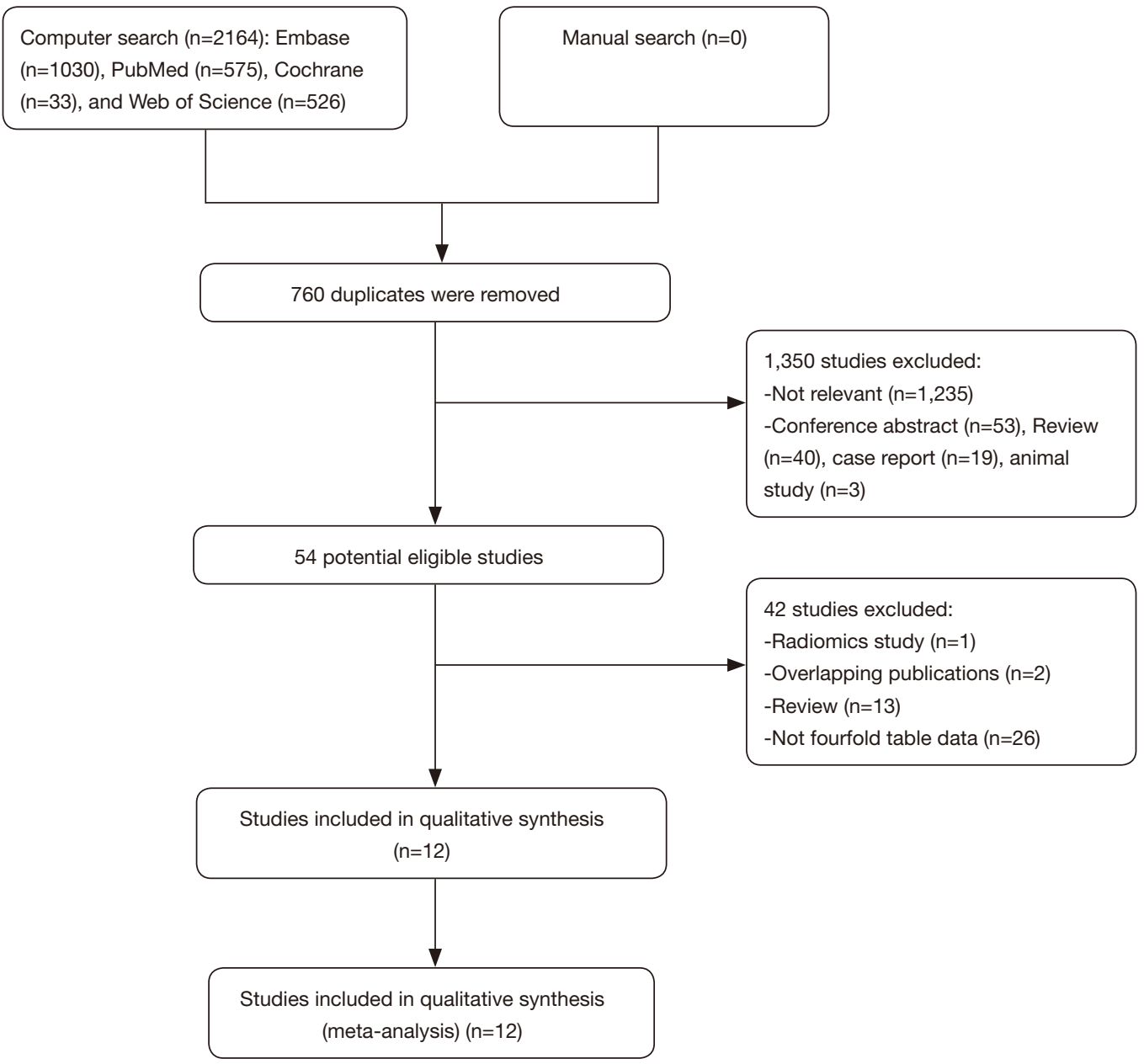

Figure 1 The diagram of the study selection process.

\section{T stage assessment}

\section{Heterogeneity test}

The chi-squared value of the studies that detected $\mathrm{T}$ stage was $9.09(\mathrm{P}=0.005<0.05)$, and the $\mathrm{I}^{2}$ value was $77.99 \%$, thus representing high heterogeneity among the six included studies.

\section{Pooled analysis results}

The pooled analysis results for the use of 18F-FDG PET/ MRI in diagnosing breast cancer T stage were as follows: sensitivity $=91 \%$ (95\% CI: 84-96\%), specificity $=91 \%$ (95\% CI: 81-96\%), DOR =105.79 (95\% CI: 41.21-271.60), PLR $=10.10$ (95\% CI: 4.61-22.11), NLR $=0.10$ (95\% CI: $0.05-$ 0.18 ), and $\mathrm{AUC}=0.96$ (95\% CI: 0.94-0.98). The forest plots and ROC curves are shown in Figures 2,3.

\section{$N$ stage assessment}

\section{Heterogeneity test}

The four studies that evaluated $\mathrm{N}$ stage assessment had a chi-squared value of $4.81(\mathrm{P}=0.045<0.05)$ and an $\mathrm{I}^{2}$ value of $58.44 \%$, demonstrating moderate heterogeneity for $\mathrm{N}$ stage assessment.

\section{Pooled analysis results}

The sensitivity, specificity, DOR, PLR, NLR, and AUC of $18 \mathrm{~F}-\mathrm{FDG}$ PET/MRI in evaluating the $\mathrm{N}$ stage of breast cancer were 94\% (95\% CI: 83-98\%), 90\% (95\% CI: 81-95\%), 130.01 (95\% CI: 48.96-345.22), 9.22 (95\% CI: 4.98-17.07), 0.07 (95\% CI: 0.03-0.19), and 0.96 (95\% CI: 0.94-0.97), respectively, which are presented in Figures 2,3. 
Table 1 Baseline characteristics of the included studies

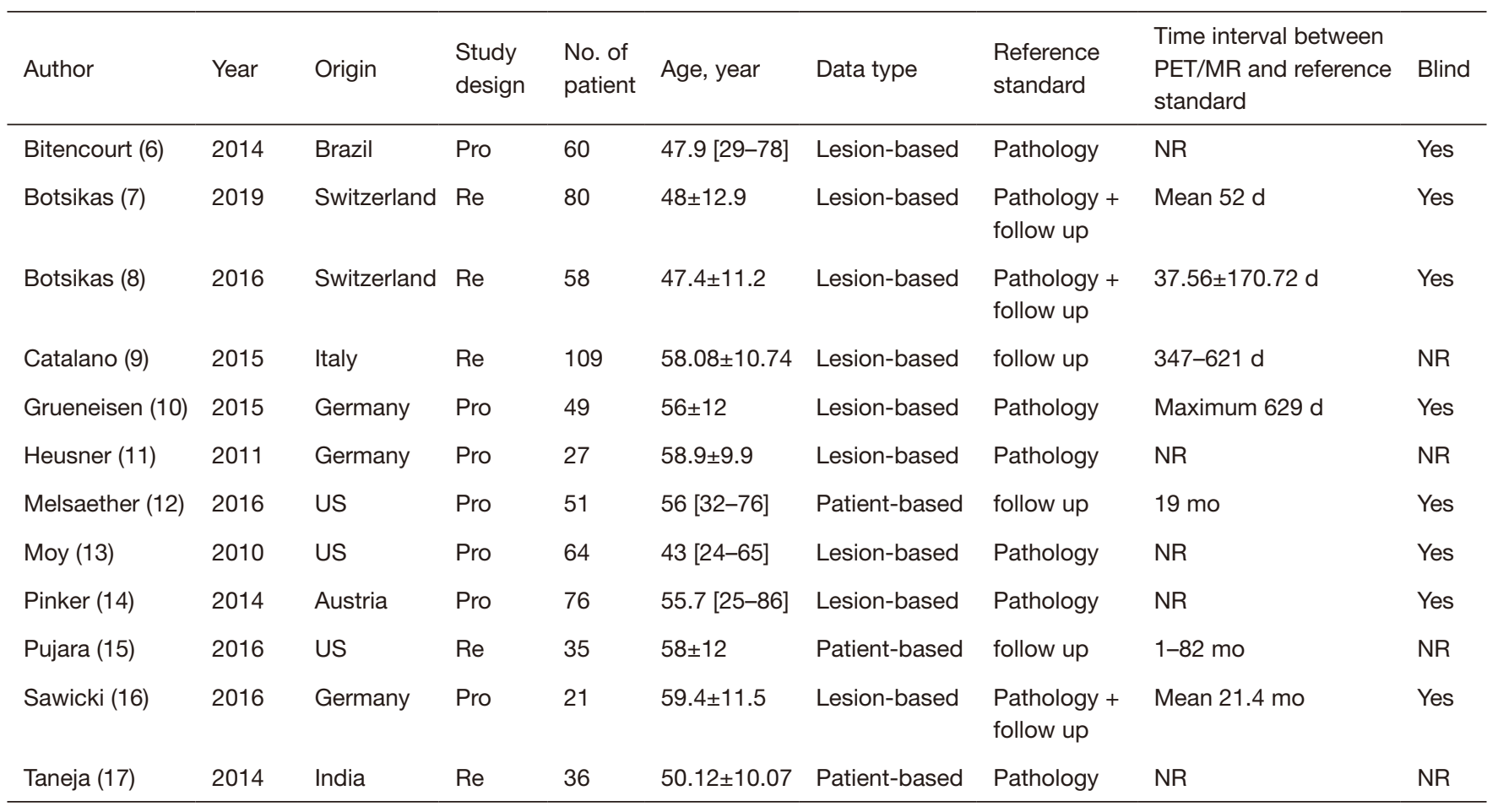

No., number; Pro, prospective; Re, retrospective; NR, not report.

Table 2 The methodological quality distribution of the included studies according to the QUADAS-2 tool

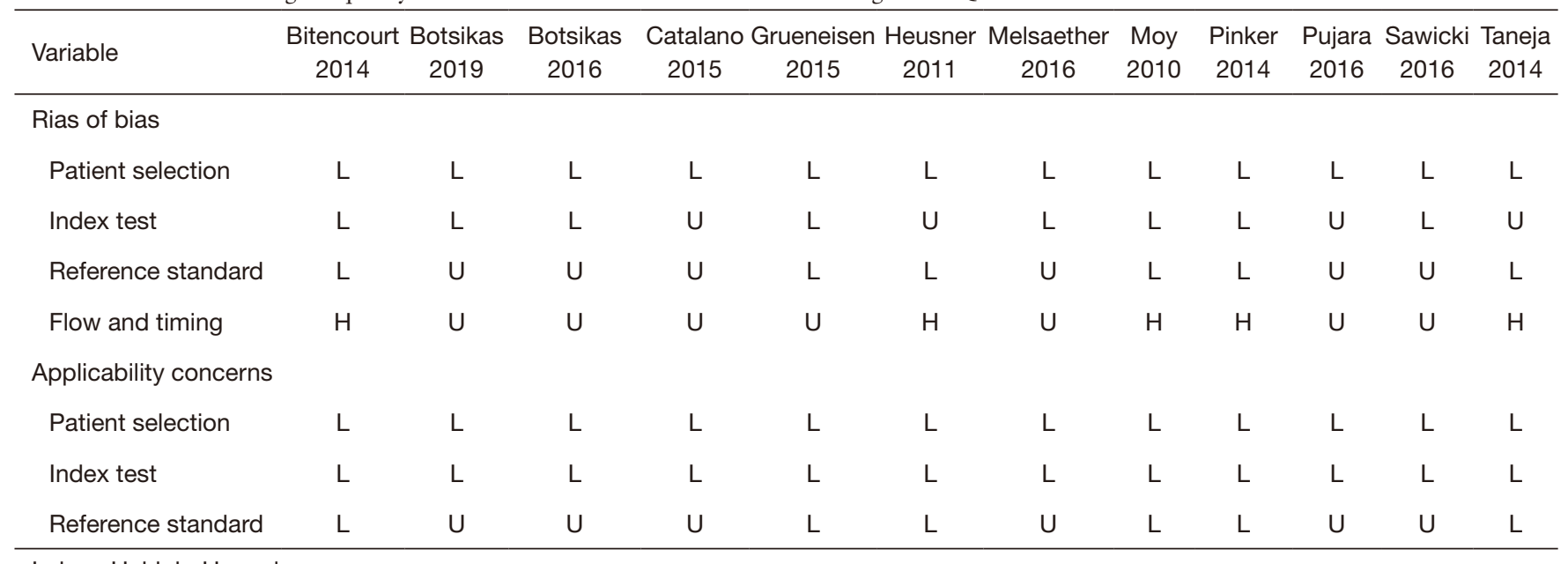

L, low; $\mathrm{H}$, high; $\mathrm{U}$, unclear.

\section{M stage assessment}

\section{Heterogeneity test}

The chi-squared value of the studies that detected $M$ stage was $8.56(\mathrm{P}=0.007<0.05)$, and the $\mathrm{I}^{2}$ value was $76.63 \%$, representing high heterogeneity among the five included studies.

\section{Pooled analysis results}

The pooled results of 18F-FDG PET/MRI in detecting breast cancer $M$ stage were as follows: sensitivity $=98 \%(95 \%$ CI: 96-99\%), specificity $=96 \%$ (95\% CI: $83-99 \%$ ), DOR 
T-stage

\begin{tabular}{|c|c|c|c|c|c|c|c|}
\hline Study & TP & FP & FN & TN & Sensitivity (95\% Cl) & Specificity $(95 \% \mathrm{CI})$ & Sensitivity $(95 \% \mathrm{Cl})$ \\
\hline Bitencour t2014 & 53 & 4 & 6 & 13 & $0.90[0.79,0.96]$ & $0.76[0.50,0.93]$ & \\
\hline Botsikas 2016 & 64 & 0 & 19 & 18 & $0.77[0.67,0.86]$ & $1.00[0.81,1.00]$ & \\
\hline Grueneisen 2015 & 57 & 3 & 4 & 19 & $0.93[0.84,0.98]$ & $0.86[0.65,0.97]$ & \\
\hline Heusner 2011 & 38 & 4 & 5 & 11 & $0.88[0.75,0.96]$ & $0.73[0.45,0.92]$ & \\
\hline Melsaether 2016 & 17 & 1 & 0 & 33 & $1.00[0.80,1.00]$ & $0.97[0.85,1.00]$ & $\rightarrow$ \\
\hline Moy 2010 & 48 & 1 & 8 & 33 & $0.86[0.74,0.94]$ & $0.97[0.85,1.00]$ & \\
\hline Pinker 2014 & 53 & 4 & 0 & 20 & $1.00[0.93,1.00]$ & $0.83[0.63,0.95]$ & 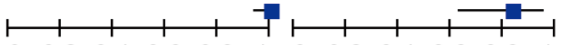 \\
\hline \multicolumn{8}{|l|}{$\mathrm{N}$-stage } \\
\hline Study & TP & FP & FN & TN & Sensitivity $(95 \% \mathrm{Cl})$ & Specificity $(95 \% \mathrm{Cl})$ & Sensitivity $(95 \% \mathrm{Cl})$ \\
\hline Botsikas 2019 & 324 & 12 & 8 & 56 & $0.98[0.95,0.99]$ & $0.82[0.71,0.91]$ & 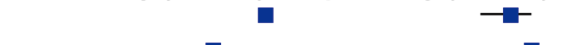 \\
\hline Grueneisen 2015 & 14 & 3 & 4 & 28 & $0.78[0.52,0.94]$ & $0.90[0.74,0.98]$ & \\
\hline Melsaether 2016 & 8 & 2 & 0 & 41 & $1.00[0.63,1.00]$ & $0.95[0.84,0.99]$ & $\rightarrow$ \\
\hline Taneja 2014 & 14 & 1 & 1 & 10 & $0.93[0.68,1.00]$ & $0.91[0.59,1.00]$ & 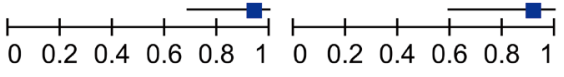 \\
\hline \multicolumn{8}{|l|}{ M-stage } \\
\hline Study & TP & FP & FN & TN & Sensitivity $(95 \% \mathrm{Cl})$ & Specificity $(95 \% \mathrm{Cl})$ & Sensitivity $(95 \% \mathrm{Cl})$ \\
\hline Botsikas 2019 & 142 & 7 & 2 & 24 & $0.99[0.95,1.00]$ & $0.77[0.59,0.90]$ & 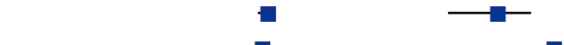 \\
\hline Catalano 2015 & 136 & 1 & 5 & 83 & $0.96[0.92,0.99]$ & $0.99[0.94,1.00]$ & + \\
\hline Melsaether 2016 & 30 & 2 & 0 & 19 & $1.00[0.88,1.00]$ & $0.90[0.70,0.99]$ & $\rightarrow$ \\
\hline Pujara 2016 & 21 & 0 & 0 & 14 & $1.00[0.84,1.00]$ & $1.00[0.77,1.00]$ & $\square$ \\
\hline Sawicki 2016 & 116 & 0 & 2 & 16 & $0.98[0.94,1.00]$ & $1.00[0.79,1.00]$ & $1 \frac{1}{1} \quad 1$ \\
\hline
\end{tabular}

Figure 2 Forest plots of pooled sensitivity and specificity for 18F-FDG PET/MRI in breast cancer TNM staging. 18F-FDG PET/MRI, 18F-fluorodeoxyglucose positron emission tomography/magnetic resonance imaging; TNM, tumor-node-metastasis.
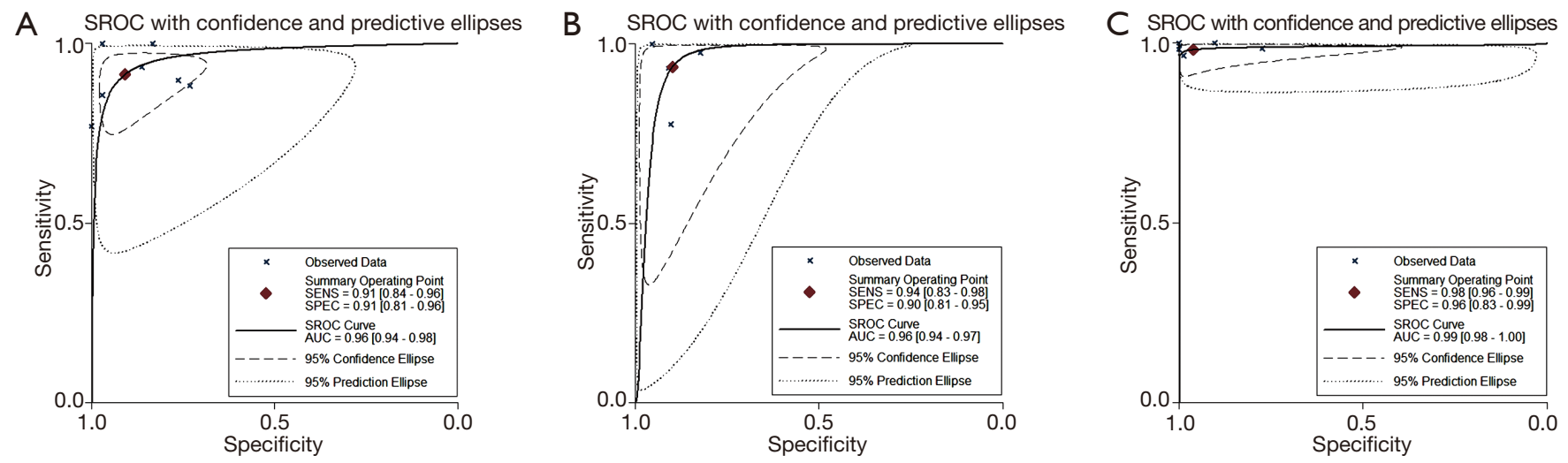

Figure 3 Forest plots of ROC for TNM staging ROC analysis of 18F-FDG PET/MRI in T stage diagnosis (A), N stage evaluation (B), and $M$ stage detection (C) in breast cancer. ROC, receiver operating characteristic; 18F-FDG PET/MRI, 18F-fluorodeoxyglucose positron emission tomography/magnetic resonance imaging; TNM, tumor-node-metastasis.

$=1,273.84$ (95\% CI: 261.14-6,213.77), PLR =25.32 (95\%

CI: 5.29-121.24), NLR $=0.02$ (95\% CI: 0.01-0.03), and AUC $=0.99$ (95\% CI: 0.98-1.00). The forest plots and ROC curves are shown in Figures 2,3.

\section{Analysis of sensitivity and publication bias}

For evaluating the reliability and stability of the pooled results, sensitivity analysis was performed by removing one study (12) with patient-based data type in T stage 

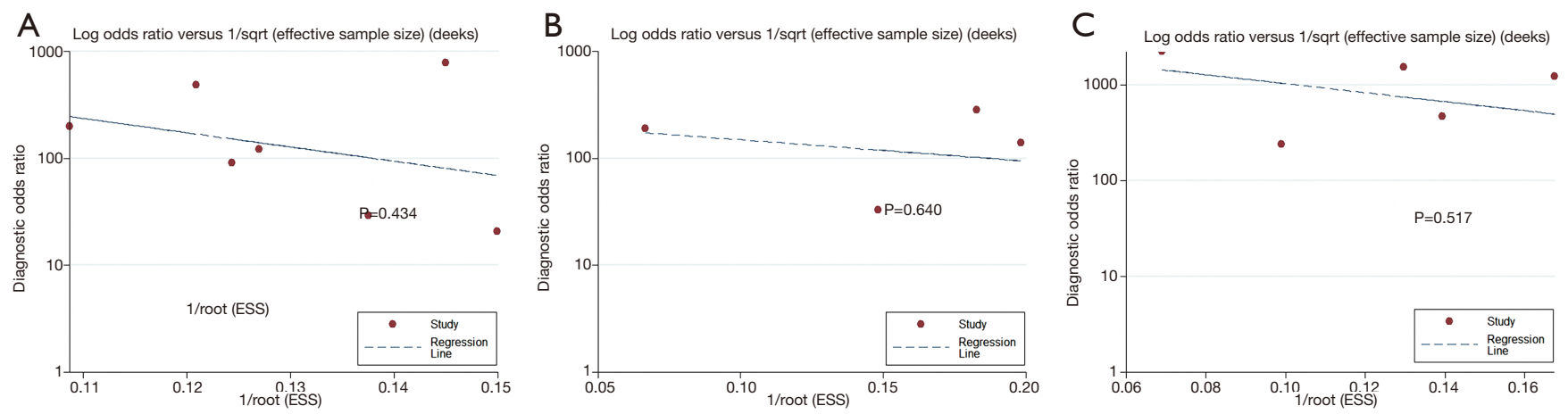

Figure 4 Deeks' funnel plot for TNM staging. Deeks' funnel plot for publication bias assessment of 18F-FDG PET/MRI for the diagnosis of $\mathrm{T}$ stage (A), the evaluation of $\mathrm{N}$ stage (B), and the detection of $\mathrm{M}$ stage (C) in breast cancer. 18F-FDG PET/MRI, 18F-fluorodeoxyglucose positron emission tomography/magnetic resonance imaging; TNM, tumor-node-metastasis.

assessment. The resulting sensitivity, specificity and AUC value was 0.90 (95\% CI: 0.83-0.95), SPE was 0.89 (95\% CI: 0.78-0.95) and AUC was 0.95 (95\% CI: 0.93-0.97), which indicated that the effect values of the sensitivity analysis were still within the $95 \% \mathrm{CI}$ of the original pooled result, thus showing that the pooled results of T-stage assessment were reliable and stable. Deeks' funnel plots are shown in Figure 4 and revealed no obvious publication bias for 18F-FDG PET/MRI in T stage diagnosis $(\mathrm{P}=0.434>0.05)$, $\mathrm{N}$ stage evaluation $(\mathrm{P}=0.640>0.05)$, or $\mathrm{M}$ stage detection $(\mathrm{P}=0.517>0.05)$.

\section{Discussion}

In recent decades, progress in preoperative diagnosis and therapy strategies has contributed to decreased mortality and increased survival in breast cancer patients. However, optimal treatment selection and patient prognosis prediction are fundamentally based on tumor location and tumor extent (19). Thus, precise TNM staging of breast cancer is not only crucial for diagnosis but is also important for guiding subsequent treatment. Ultrasound is routinely used for local tumor diagnosis and lymph node evaluation with the advantage of multiangle real-time scanning and tissue flow analysis, but ultrasound is not recommended for detecting distant metastasis. In addition, the relatively low sensitivity of ultrasound for detecting breast cancer (20) and evaluating (21) lymph node metastasis $(80.1 \%$ and $69.4 \%$, respectively) limits its clinical utility.

18F-FDG PET/CT is an integrated imaging technique that is commonly performed for initial TNM staging and neoadjuvant therapy assessment in breast cancer patients (22). However, 18F-FDG PET/CT demonstrates a high radiation dose and low sensitivity of 0.56 (95\% CI: $0.47-0.63)$ for detecting lymph node metastases (23) and is also limited in detecting small and low-grade lesions owing to the low degree of glycolytic metabolism and low softtissue contrast of CT (24). In contrast, Hong et al. (25) demonstrated that the diagnostic performance of $18 \mathrm{~F}-\mathrm{FDG}$ $\mathrm{PET} / \mathrm{CT}$ is comparable to that of bone scintigraphy, with a sensitivity of 0.97 (95\% CI: $0.84-0.99)$ and specificity of 0.95 (95\% CI: 0.93-0.97) in detecting bone metastases. Compared to ultrasound and 18F-FDG PET/CT imaging techniques, the high spatial resolution and soft-tissue contrast provided with MRI (26) contribute to improved diagnostic value not only in breast cancer but also in regional lymph nodes $(27,28)$, with reported sensitivities of 0.92 (95\% CI: $0.89-0.94)$ and 0.55 (95\% CI: 0.48-0.62), respectively. However, the MRI technique has not been confirmed to be accurate as a potential alternative to detect distant metastases (29).

18F-FDG PET/MRI is a fusion imaging modality that combines the functional information of FDG PET and the morphological data of MRI in one examination, thus demonstrating well-accepted diagnostic and staging value in the preoperative assessment of primary and metastatic cancers, such as lymphomas (30), head and neck tumors (31), and liver cancer (32). In this meta-analysis, 18F-FDG PET/ MRI demonstrated sensitivity and specificity values of $91 \%$ (95\% CI: 84-96\%) and 91\% (95\% CI: 81-96\%), 94\% (95\% CI: $83-98 \%$ ) and $90 \%$ (95\% CI: 81-95\%), and 98\% (95\% CI: $96-99 \%)$ and 96\% (95\% CI: 83-99\%) for T stage, $\mathrm{N}$ stage and $\mathrm{M}$ stage, respectively, indicating that $18 \mathrm{~F}-\mathrm{FDG}$ PET/MRI may serve as a promising imaging examination 
method for TNM staging in breast cancer. The study by Lin et al. presented different sensitivity values [from 0.91 (95\% CI: $0.88-0.94)$ to 0.98 (95\% CI: $0.95-0.99$ )] and specificity values [from 0.87 (95\% CI: $0.76-0.95$ ) to 0.95 (95\% CI: 0.92-0.97)] for 18F-FDG PET/MRI in wholebody breast cancer staging/restaging. In our meta-analysis, the pooled sensitivity and specificity of 18F-FDG PET/ MRI in TNM staging were different from the results of Lin et al. (18), suggesting that TNM staging could provide more precise value for staging breast cancer than wholebody staging. Furthermore, five original studies neglected in their study $(6,7,11,13,14)$ but included in our study may also account for this difference between TNM staging and whole-body staging.

Compared to single $18 \mathrm{~F}-\mathrm{FDG} \mathrm{PET} / \mathrm{CT}$ or MRI techniques, fusion 18F-FDG PET/MRI tends to perform better in breast cancer TNM staging, which is in agreement with the previous conclusion of Taneja et al. (17). Two factors may contribute to this result: (I) Breast cancer presents high glucose metabolism and glycolysis rates within the mammary gland, which is filled with soft tissue; these features indicate $18 \mathrm{~F}$-FDG PET/MRI imaging as an appropriate method for breast cancer diagnosis and lymph node evaluation, as MRI has excellent soft-tissue contrast (33), and the diagnostic ability of MRI may be improved by combining the imaging features of MRI with the functional information of FDG PET. Additionally, quantitative parameter from PET/MRI were non-invasive prognostic markers in the breast cancer (34). (II) Standard uptake values (SUVs) are not reliable as imaging biomarkers of distant metastasis on FDG PET , but malignancies with faint radiotracer uptake that are invisible on PET can be effectively diagnosed with reference to MRI images, owing to the delayed acquisition time of MRI compared with reduced background uptake and increased lesion visibility (35), especially for small lytic or permeative bony lesions.

This meta-analysis demonstrates that the combined analysis of 18F-FDG PET/MRI improves the diagnostic accuracy of breast cancer TNM staging; however, neither the sensitivity nor specificity reached $100 \%$. Based on our clinical experience and previous studies, some of the following potential factors could account for FP or FN results: (I) PET and MRI images are limited by the low spatial resolution, as the detection rates decline with decreasing tumor size (24) and is especially limited for small tumors $(<10 \mathrm{~mm})$. (II) Tumor grade and histopathology are important factors affecting FDG uptake (36), as high-grade and infiltrating ductal breast cancers usually present high FDG uptake values, thus making the diagnosis of low-grade and noninfiltrating ductal breast cancers difficult. (III) As N stage depends on the number of infiltrated lymph nodes, the differences between radiological $\mathrm{N}$ stage and pathological $\mathrm{N}$ stage can probably be explained by the fact that the number of infiltrated lymph nodes reported on PET/MR was different from that found on pathological examination, which can detect small lymph node metastases not found by imaging. (IV) Benign bone changes (osteoarthritis, osseous infarctions and vertebral hemangiomas) and magnetic field inhomogeneities resulting from air artifacts may hamper MRI diagnostic performance (37).

There were several limitations that should be analyzed when interpreting the results of this meta-analysis. First, study methodological characteristics, including study design and data type, may contribute to high heterogeneity and produce a negative impact on the pooled results for 18F-FDG PET/MRI in TNM staging. However, further subgroup analysis was unable to be performed because of the limited number of enrolled studies and extracted data (Table 1). Second, only twelve studies with 666 patients were finally included in this meta-analysis, and both computer and manual search strategies were adopted; therefore, high-quality studies with larger sample sizes are required to confirm the diagnostic value of $18 \mathrm{~F}-\mathrm{FDG}$ PET/MRI in the TNM staging of breast cancer. Third, the exclusion of review articles, conference abstracts and studies published in non-English languages may lead to potential publication bias. Additionally, although pathological results are the reference standard for breast cancer diagnosis and staging, histological diagnosis is not available for breast cancer patients with distant metastasis proved by imaging diagnosis, and follow-up imaging criteria are sufficiently accurate for breast cancer staging.

In conclusion, these meta-analysis results reveal that 18F-FDG PET/MRI provides high diagnostic accuracy in diagnosing local tumor invasion, evaluating regional lymph node involvement, and detecting distant metastases in breast cancer, indicating that $18 \mathrm{~F}-\mathrm{FDG}$ PET/MRI may serve as a valuable and promising imaging technique for TNM staging in breast cancer.

\section{Acknowledgments}

Funding: This work was supported by the Lanzhou Talent Innovation and Entrepreneurship Project Foundation of China (No. 2017-RC-76). 


\section{Footnote}

Reporting Checklist: The authors have completed the PRISMA reporting checklist. available at: http://dx.doi. org/10.21037/apm-20-2555

Conflicts of Interest: All authors have completed the ICMJE uniform disclosure form (available at: http://dx.doi. org/10.21037/apm-20-2555). The authors have no conflicts of interest to declare.

Ethical Statement: The authors are accountable for all aspects of the work in ensuring that questions related to the accuracy or integrity of any part of the work are appropriately investigated and resolved.

Open Access Statement: This is an Open Access article distributed in accordance with the Creative Commons Attribution-NonCommercial-NoDerivs 4.0 International License (CC BY-NC-ND 4.0), which permits the noncommercial replication and distribution of the article with the strict proviso that no changes or edits are made and the original work is properly cited (including links to both the formal publication through the relevant DOI and the license). See: https://creativecommons.org/licenses/by-nc-nd/4.0/.

\section{References}

1. Bray F, Ferlay J, Soerjomataram I, et al. Global cancer statistics 2018: GLOBOCAN estimates of incidence and mortality. CA Cancer J Clin 2018;68:394-424.

2. Momenimovahed Z, Salehiniya H. Epidemiological characteristics of and risk factors for breast cancer in the world. Breast Cancer 2019;11:151-64.

3. Gradishar WJ, Anderson BO, Balassanian R, et al. Breast Cancer, Version 4.2017, NCCN Clinical Practice Guidelines in Oncology. J Natl Compr Canc Netw 2018;16:310-20.

4. DeSantis CE, Lin CC, Mariotto AB, et al. Cancer treatment and survivorship statistics 2014. CA Cancer J Clin 2014;64:252-71.

5. Giuliano AE, Connolly JL, Edge SB, et al. Breast CancerMajor changes in the American Joint Committee on Cancer eighth edition. CA Cancer J Clin 2017;67:290-30.

6. Bitencourt AG, Lima EN, Chojniak R, et al. Can 18F-FDG PET improve the evaluation of suspicious breast lesions on MRI? Eur J Radiol 2014;83:1381-6.

7. Botsikas D, Bagetakos I, Picarra M, et al. What is the diagnostic performance of 18-FDG-PET/MR compared to PET/CT for the $\mathrm{N}$ - and $\mathrm{M}$ - staging of breast cancer? Eur Radiol 2019;29:1787-98.

8. Botsikas D, Kalovidouri A, Becker M, et al. Clinical utility of $18 \mathrm{~F}-\mathrm{FDG}-\mathrm{PET} / \mathrm{MR}$ for preoperative breast cancer staging. Eur Radiol 2016;26:2297-307.

9. Catalano OA, Nicolai E, Rosen BR, et al. Comparison of CE-FDG-PET/CT with CE-FDG-PET/MR in the evaluation of osseous metastases in breast cancer patients. Br J Cancer 2015;112:1452-60.

10. Grueneisen J, Nagarajah J, Buchbender C, et al. Positron Emission Tomography/Magnetic Resonance Imaging for Local Tumor Staging in Patients with Primary Breast Cancer: A Comparison with Positron Emission Tomography/Computed Tomography and Magnetic Resonance Imaging. Invest Radiol 2015;50:505-13.

11. Heusner TA, Hahn S, Jonkmanns C, et al. Diagnostic accuracy of fused positron emission tomography/magnetic resonance mammography: Initial results. Br J Radiol 2011;84:126-35.

12. Melsaether AN, Raad RA, Pujara AC, et al. Comparison of whole-body 18F FDG PET/MR imaging and wholebody 18F FDG PET/CT in terms of lesion detection and radiation dose in patients with breast cancer. Radiology 2016;281:193-202.

13. Moy L, Noz ME, Maguire GQ, et al. Role of fusion of prone FDG-PET and magnetic resonance imaging of the breasts in the evaluation of breast cancer. Breast J 2010;16:369-76.

14. Pinker K, Bogner W, Baltzer P, et al. Improved differentiation of benign and malignant breast tumors with multiparametric 18 fluorodeoxyglucose positron emission tomography magnetic resonance imaging: A feasibility study. Clin Cancer Res 2014;20:3540-9.

15. Pujara AC, Raad RA, Ponzo F, et al. Standardized uptake values from PET/MRI in metastatic breast cancer: An organ-based comparison with PET/CT. Breast J 2016;22:264-73.

16. Sawicki LM, Grueneisen J, Schaarschmidt BM, et al. Evaluation of 18F-FDG PET/MRI, 18F-FDG PET/CT, MRI, and CT in whole-body staging of recurrent breast cancer. Eur J Radiol 2016;85:459-65.

17. Taneja S, Jena A, Goel R, et al. Simultaneous whole-body ${ }^{18} \mathrm{~F}$-FDG PET-MRI in primary staging of breast cancer: a pilot study. Eur J Radiol 2014;83:2231-9.

18. Lin CY, Lin CL, Kao CH. Staging/restaging performance of F18-fluorodeoxyglucose positron emission tomography/ magnetic resonance imaging in breast cancer: A review and 
meta-analysis. Eur J Radiol 2018;107:158-65.

19. Wörmann B. Breast cancer: basics, screening, diagnostics and treatment. Med Monatsschr Pharm 2017;40:55-64.

20. Sood R, Rositch AF, Shakoor D, et al. Ultrasound for Breast Cancer Detection Globally: A Systematic Review and Meta-analysis. J Glob Oncol 2019;5:1-17.

21. Zhang YN, Wang CJ, Xu Y, et al. Sensitivity, Specificity and Accuracy of Ultrasound in Diagnosis of Breast Cancer. Ultrasound Med Biol 2015;41:1835-41.

22. Caresia Aroztegui AP, García Vicente AM, Alvarez Ruiz S, et al. 18F-FDG PET/CT in breast cancer: Evidencebased recommendations in initial staging. Tumour Biol 2017;39:1010428317728285.

23. Zhang X, Liu Y, Luo H, et al. PET/CT and MRI for Identifying Axillary Lymph Node Metastases in Breast Cancer. J Magn Reson Imaging 2020;52:1840-51.

24. Koolen BB, van der Leij F, Vogel WV, et al. Accuracy of 18F-FDG PET/CT for primary tumor visualization and staging in T1 breast cancer. Acta Oncol 2014;53:50-7.

25. Hong S, Li J, Wang S. 18FDG PET-CT for diagnosis of distant metastases in breast cancer patients. A metaanalysis. Surg Oncol 2013;22:139-43.

26. Choi YJ, Lee IS, Song YS, et al. Diagnostic performance of diffusion-weighted (DWI) and dynamic contrastenhanced (DCE) MRI for the differentiation of benign from malignant soft-tissue tumors. J Magn Reson Imaging 2019;50:798-809.

27. Zhang Y, Ren H. Meta-analysis of diagnostic accuracy of magnetic resonance imaging and mammography for breast cancer. J Cancer Res Ther 2017;13:862-8.

28. Zhou P, Wei Y, Chen G, et al. Axillary lymph node metastasis detection by magnetic resonance imaging in

Cite this article as: $\mathrm{Lu} \mathrm{XR,} \mathrm{Qu} \mathrm{MM,} \mathrm{Zhai} \mathrm{YN,} \mathrm{Feng} \mathrm{W,} \mathrm{Gao} \mathrm{Y,}$ Lei JQ. Diagnostic role of 18F-FDG PET/MRI in the TNM staging of breast cancer: a systematic review and meta-analysis. Ann Palliat Med 2021;10(4):4328-4337. doi: 10.21037/apm-20-2555 patients. Thorac Cancer 2018;9:989-996.

29. Wu LM, Gu HY, Zheng J, et al. Diagnostic value of whole-body magnetic resonance imaging for bone metastases: a systematic review and meta-analysis. J Magn Reson Imaging 2011;34:128-35.

30. Schwenzer NF, Pfannenberg AC. PET/CT, MR, and PET/MR in Lymphoma and Melanoma. Semin Nucl Med 2015;45:322-31.

31. Huellner MW. PET/MR in Head and Neck Cancer-An Update. Semin Nucl Med 2021;51:26-38.

32. Lee DH, Lee JM, Hur BY, et al. Colorectal Cancer Liver Metastases: Diagnostic Performance and Prognostic Value of PET/MR Imaging. Radiology 2016;280:782-92.

33. Boss A, Bisdas S, Kolb A, et al. Hybrid PET/MRI of intracranial masses: initial experiences and comparison to PET/CT. J Nucl Med 2010;51:1198-205.

34. Gelezhe PB, Blokhin IA, Marapov DI, et al. Quantitative parameters of MRI and 18 F-FDG PET/CT in the prediction of breast cancer prognosis and molecular type: an original study. Am J Nucl Med Mol Imaging 2020;10:279-92.

35. Catalano OA, Rosen BR, Sahani DV, et al. Clinical impact of PET/MR imaging in patients with cancer undergoing same-day PET/CT: initial experience in 134 patients-a hypothesis-generating exploratory study. Radiology 2013;269:857-69.

36. Koo HR, Park JS, Kang KW, et al. 18F-FDG uptake in breast cancer correlates with immunohistochemically defined subtypes. Eur Radiol 2014;24:610-8.

37. Shikhare S, Chacko JK, Chuah KL. Regional bone change in intramuscular haemangioma mimicking primary bone tumour. J Med Imaging Radiat Oncol 2015;59:204-6. 